Research Paper

\title{
Salmonella typhimurium Mediated Delivery of Apoptin in Human Laryngeal Cancer
}

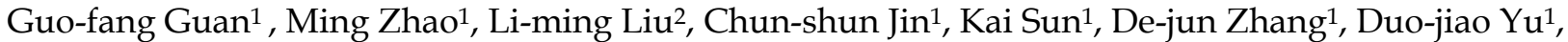 \\ Hong-wei $\mathrm{Cao}^{1}$, Yan -qing Lu ${ }^{1}$ and Lian-ji Wen ${ }^{\circledR}$ \\ 1. Department of Otolaryngology, Head and Neck Surgery, The Second Hospital of Jilin University, Changchun 130041, P. R. China \\ 2. Jilin Academy of Agricultural Science and Technology, Jilin 132101, P. R. China
}

$\triangle$ Corresponding author: Dr. Lian-ji Wen. Department of Otolaryngology Head and Neck Surgery, The Second Hospital of Jilin University, No. 218, Zhiqiang Street, Changchun 130041, P. R. China. Tel +86 43188796636 . Fax +86 431 88934741. Email : lianjiwen1@gmail.com

(c) Ivyspring International Publisher. This is an open-access article distributed under the terms of the Creative Commons License (http://creativecommons.org/ licenses/by-nc-nd/3.0/). Reproduction is permitted for personal, noncommercial use, provided that the article is in whole, unmodified, and properly cited.

Received: 2013.06.20; Accepted: 2013.08.30; Published: 2013.09.2I

\begin{abstract}
An effective cancer therapeutic should target tumours specifically with limited systemic toxicity. Here, we transformed an attenuated Salmonella typhimurium (S. typhimurium) with an Apoptin expressing plasmid into a human laryngeal carcinoma cell line. The expression of the inserted gene was measured using fluorescence and immunoblotting assays. The attenuated S. typhimurium-mediated Apoptin significantly decreased cytotoxicity and strongly increased cell apoptosis through the activation of caspase-3. The process was mediated by Bax, cytochrome $c$ and caspase-9. A syngeneic nude murine tumour model was used to determine the anti-tumour effects of the recombinant bacteria in vivo. Systemic injection of the recombinant bacteria with and without re-dosing caused significant tumour growth delay and reduced tumour microvessel density, thereby extending host survival. Our findings indicated that the use of recombinant Salmonella typhimurium as an Apoptin expression vector has potential cancer therapeutic benefits.
\end{abstract}

Key words: tumours specifically therapy; attenuated Salmonella typhimurium; laryngeal cancer; Apoptin; apoptosis

\section{Introduction}

Head and neck squamous carcinoma is estimated to be the sixth most common malignant tumour worldwide [1], of which laryngeal carcinoma is the second most common type [2]. This carcinoma has a poor prognosis due to the fact that it doesn't usually cause symptoms until it is too late, leading to low treatment efficacy and high recurrence rate [3]. The incidence and mortality rates of laryngeal carcinoma are equivalent, which suggests a failure of current therapies, despite of the variety of combined modality approaches that have been introduced to complement surgical treatment $[4,5]$.

Many bacteria have been shown to selectively target tumours and have been used in cancer gene delivery $[6,7]$. However, once these bacteria are rendered non-toxic to normal human cells, their toxicity against cancer cells is also reduced [8]. To produce significant anti-tumour responses, high concentrations and multiple administrations of the bacteria are often required [9]. Such regimens however, may cause damage to normal tissues [10]. The current state of art by using bacterial therapies has highlighted the need for novel strategies to induce effective responses in clinical trials. Genetic manipulation is one of the most promising strategies to enhance bacterial delivery.

Salmonella typhimurium (S. typhimurium), a facultative anaerobe, has already been utilized as an anti-cancer agent and has been shown to selectively target tumours after intravenous injection [11]. $S$. typhimurium has particular promise as a cancer therapeutic agent because it can be administrated systemically in its active form, which permits microbes to penetrate and traverse tumour tissue to target specific microenvironments [9, 12]. Furthermore, S. typhi- 
murium preferentially accumulates in tumours more than other organs after systemic injection, which reduces off-target bacterial toxicity $[9,13]$. S. typhimurium can be also used to treat tumours that are p53-deficient or resistant to chemotherapeutic and radiation therapies $[9,14,15]$.

A chicken anemia virus derived protein, Apoptin, is a powerful potential agent for cancer gene therapy and has been demonstrated to selectively induce apoptosis in a large panel of human malignant cells, whereas normal cells remain unaffected [16-18]. The excellent efficacy and safety of Apoptin in cancer-specific therapeutics have been reported in several previous studies in various ways [18-20].

Here, we present an approach incorporating the delivery of the cancer-specific cytotoxic protein, Apoptin, by using attenuated S. typhimurium as a vector in human laryngeal cancer. When systemically delivered into mice, attenuated $S$. typhimurium-mediated Apoptin was capable of inducing apoptosis, causing significant tumour regression while being associated with minimal systemic toxicity.

\section{Materials and Methods}

\section{Cell line, bacteria, plasmids and animals}

The human laryngeal cancer cell line, Hep-2, was purchased from the China Center for Type Culture Collection and maintained at $37^{\circ} \mathrm{C}, 5 \% \mathrm{CO}_{2}$ in RPMI 1640 complete medium (Gibco, USA) with $10 \%$ fetal bovine serum (FBS, Gibco, USA). The attenuated $S$. typhimurium phoP/phoQ null strain LH430 [21] was a kind gift from Prof. Xue-jian Zhao of Jilin University of China. Escherichia coli (E. coli) DH5a, restriction endonucleases and plasmid pcDNA3.1 were purchased from Takara (Shiga, Japan). The pGH-Apoptin and pGH-EGFP plasmids were obtained from Sangon (China). Six to eight week old female BALB/c nude mice were obtained from the Shanghai Institute of Experimental Animals (China).

\section{Construction of expression vectors}

Prokaryotic expression plasmids were generated that contained Apoptin or enhanced green fluorescent protein (EGFP) under the control of the cytomegalovirus (CMV) promoter. In brief, pcDNA-EGFP, the control plasmid, was constructed by excising the EGFP fragment from pGH-EGFP using EcoRI and PstI, and sub-cloned into EcoRI and PstI sites on pcDNA3.1. The pcDNA-Apoptin construct was developed by sub-cloning the EcoRI/PstI-digested Apoptin fragment from pGH-Apoptin into pcDNA3.1. The appropriate sequences of inserts were confirmed with use of an ABI DNA Sequencer (USA).
Attenuated S. typhimurium transformation using electroporation

The attenuated S. typhimurium strain, LH430, was harvested at $4^{\circ} \mathrm{C}$ and washed in ice-cold $10 \%$ glycerol. Then, the bacteria were re-suspended into electrotransfer buffer ( $10 \%$ glycerol, $0.125 \%$ yeast extract, $0.25 \%$ tryptone) and electro-transferred into 0.2 $\mathrm{cm}$ cuvettes after $40 \mu \mathrm{l}$ competent cells were mixed with either $25 \mathrm{ng}$ plasmid DNA pcDNA-EGFP or pcDNA-Apoptin using a BTX ECM399 electroporation system (USA). The recombinant attenuated $S$. typhimurium strains were then selected and maintained in Luria-Bertani (LB) media with $50 \mu \mathrm{g} / \mathrm{ml}$ ampicillin. Bacteria electroporated with pcDNA-EGFP or pcDNA-Apoptin plasmid constructs were named ST-rC-EGFP and ST-rC-Apoptin, respectively.

\section{Cell infection and western blotting}

Cell infection, cell lysis, protein quantification, and western blots were performed as described previously [21, 22]. In brief, Hep-2 cells $\left(2 \times 10^{5}\right)$ were co-cultured with recombinant bacteria $\left(1 \times 10^{8} \mathrm{cfu}\right)$ for 1 hour at $37^{\circ} \mathrm{C}$. The infected cells were then rinsed and cultured in RPMI 1640 medium containing gentamycin sulfate $(20 \mu \mathrm{g} / \mathrm{ml})$ to eliminate external bacteria and tetracycline $(5 \mu \mathrm{g} / \mathrm{ml})$ to prevent infection with internal bacteria. Twenty-four hours after infections, the cells were collected and lysed. Protein extracts were prepared and quantified using the BCA method [22]. With $20 \mu \mathrm{g}$ of protein per well, 15\% SDS-PAGE was performed, and samples were then transferred onto Hybond-C membranes (Amersham, USA). Membranes were blocked in 5\% milk in Tris-buffered saline with $0.1 \%$ Tween-20 (TBST). Blots were incubated with anti-Apoptin (provided by Dr. Zhi-qiang Mi of Jilin university); anti-Bcl-2 (N-19, Santa Cruz biotechnology); anti-Bax (N-20, Santa Cruz biotechnology); anti-Caspase-9 (Human specific \#9502, Cell Signaling) and anti-Cytochrome c $(6 \mathrm{H} 2$, Santa Cruz biotechnology) for 2 hours followed by incubation with the second antibodies (Abcam, USA) labeled with horseradish peroxidase for 2 hours. Signals were visualized using the ECL Western blotting substrate kit (Pierce, USA).

\section{Cell proliferation assay}

Hep-2 cells $\left(5 \times 10^{3}\right)$ were plated in 96-well plates 24 hours before infection with the recombinant bacteria $\left(1 \times 10^{4} \mathrm{cfu}\right.$ per well) as described above. Cell viability was assessed using a 3-[4,5-dimethylthiazol-2yl]-2,5-diphenyltetrazolium bromide (MTT) staining kit (Sigma, USA) every day over a 6 day period according to the manufacturer's protocol. Briefly, cells were incubated with $5 \mathrm{~g} / \mathrm{L}$ of MTT for 4 hours. Then, 
the medium was removed, and $100 \mu \mathrm{l}$ of dimethylsulphoxide were added. The absorbance of the reaction solution was measured at $490 \mathrm{~nm}$. The cell viability was calculated as follows: $A=(1$ - absorbance of experimental group/absorbance of control group) $x$ $100 \%$.

\section{Annexin V and Flow cytometric apoptosis as- says}

Apoptosis was first visualized using an Annexin detection kit (eBioscience, USA) according to the manufacturer's protocol. In brief, Hep- 2 cells $\left(1 \times 10^{5}\right)$ were infected with the recombinant bacteria for 48 hours. The cells were then collected and re-suspended in binding buffer, followed by incubation with Annexin $\mathrm{V}$ labeled with fluorescein isothiocyanate (FITC) and propidium iodide (PI). The stained cells were visualized with a TCS SP5 laser scanning confocal microscope (Leica, Germany), and then analyzed on a FACSCaliber flow cytometer (Becton Dickinson). A total of 50,000 events were acquired on five parameters for each sample and the data was analyzed using CellQuest software.

\section{Caspase activity assay}

Caspase activity assays were performed on Hep-2 cells $\left(1 \times 10^{5}\right)$ after infection with the recombinant bacteria for 48 hours using the Caspase 3/8 assay kit (Clontech, USA), according to the manufacturer's protocol. Briefly, cell lysates were obtained from $2 \times 10^{5}$ infected cells and incubated in caspase- 3 substrate (DEVD-pNA) or caspase-8 substrate (IEDT-pNA). The plates were read at $450 \mathrm{~nm}$ and the data was analyzed.

\section{Immunohistochemistry}

Mice were treated as above and then sacrificed 48 hours after the treatment. Tumours were removed and immediately fixed in $10 \%$ buffered-formalin for paraffin embedded. Immunohistochemistry was performed using a purified rat anti-mouse CD31 antibody (1:50 dilution; BD Pharmingen) and evaluated using the Level-2 Ultra Streptavidin System (Signet Laboratories) with microwave antigen retrieval. The microvessel staining was scored semi-quantitatively in tumour areas. Five randomly selected areas for each tumour were evaluated at 200x magnification. Positive endothelial cell clusters were counted as an individual vessel. The average number of microvessels was defined as final result.

\section{Tumour growth experiments}

At 8 weeks age, BALB/c nude mice received a subcutaneous injection of $2 \times 10^{6}$ Hep- 2 cells. After 3 weeks of tumour growth, mice with tumours $<75$ $\mathrm{mm}^{3}$ at 21 days were excluded from the analysis and only the rest were randomized into three groups: A).
Experimental group which further divided into following three sub-groups: (i). Saline (mock) alone; (ii). Intravenous injection of $1 \times 10^{7} \mathrm{cfu}$ of ST-rC-EGFP. (iii). Intravenous injection of $1 \times 10^{7} \mathrm{cfu}$ of ST-rC-Apoptin. Each experiment was conducted independently two times, with total of five mice per group (three in first experiment; two in second experiment). B). Repeat treatment groups; same as above experimental groups, the mice were re-treated at 1 week after and were followed up with over 30 days for survival analysis (two independent experiments; total of five mice per group). C). Bacterial distribution and histologic analysis group $(n=5)$. The mice were sacrificed at 48 hours after the treatment and various mouse organs were collected, partial livers and also partial tumours were harvested for analysis of bacterial distribution, the rest of organs were fixed into $10 \%$ buffered formalin. All tumour volumes were measured twice a week using the following equation: (length)*(width)*(height)* $\Pi / 6$. Mice with tumour volumes exceeding $1,400 \mathrm{~mm}^{3}$ were sacrificed. Others were followed up until 32 days.

\section{Analysis of bacterial distribution}

Tissue samples were weighed and minced thoroughly, followed by homogenization. The tissue homogenates were plated onto LB agar containing ampicillin in triplicate, and the colony count was determined on the next day.

\section{Statistical analysis}

All data was expressed as the mean $\pm \mathrm{SE}$; a p-value of less than 0.05 was considered to be statistically significant. To measure the fraction of mice living for a certain amount of time after treatment, the Kaplan-Meier survival curves and the log-rank test [23] were utilized to establish the efficacy of S. typhimurium-mediated Apoptin treatment compared to the control treatment.

\section{Results}

\section{Expression of attenuated S. typhimuri- um-mediated Apoptin in Hep-2 cells}

To determine whether the attenuated Salmonella typhimurium can infect human laryngeal cancer cells, Hep-2 cells were infected with ST-rC-EGFP and were observed by fluorescence microscopy after 24 hours. Around $80 \%$ of infected cells showed positive for green fluorescence staining (Figure 1A). Western blotting analysis demonstrated that Apoptin was only expressed in the ST-rC-Apoptin infected cells but neither in non-infected cells (mock) nor in ST-rC-EGFP infected cells, suggesting that attenuated S. typhimurium carrying Apoptin has an ability to infect and express Apoptin in laryngeal cancer cell line 
as shown in Figure 1C.

\section{S. typhimurium-mediated Apoptin induces apoptosis and effectively reduces viability of Hep-2 cells}

To establish whether $S$. typhimurium-mediated Apoptin can induce apoptosis in Hep-2 cells, tumour cells were infected with ST-rC-Apoptin and were compared with un-infected cells. After 48 hours, the cells were stained with Annexin V PI. The apoptotic Hep-2 cells were positively stained with both Annexin-V (green) and PI (red), as shown in Figure 2A. Flow cytometric analysis was then conducted on Hep-2 cells, showing a total of $60 \%$ of both early and late apoptotic fractions in ST-rC-Apoptin, compared to $2.7 \%$ in mock cells (Figure $2 \mathrm{~B}$ ). The MTT assay was used to determine the cytotoxic effects of ST-rC-Apoptin on Hep-2 cells (Figure 2C). Cell viability continuously increased from day 1 to day 3 and saturated to $\sim 80 \%$ and $70 \%$ at days 4 through 6 at both mock and ST-rC-EGFP cells, respectively. However, ST-rC-Apoptin infected cells caused consistent cytotoxicity, with cell viability reducing to $10 \%$ at day 2 , although it later recovered to $30 \%$ at day 4 , cytotoxicity eventually declined over the time, reaching $36 \%$ by day 6 . The data demonstrated significant cy-

A

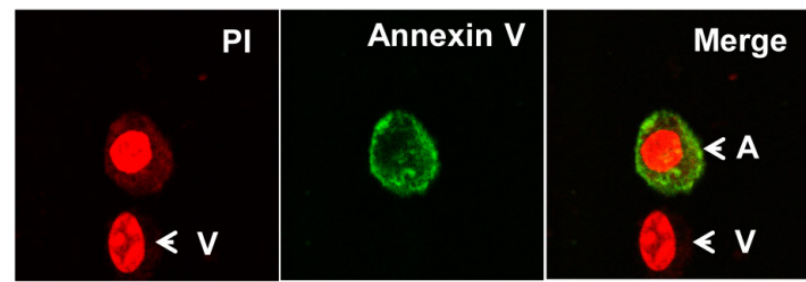

B
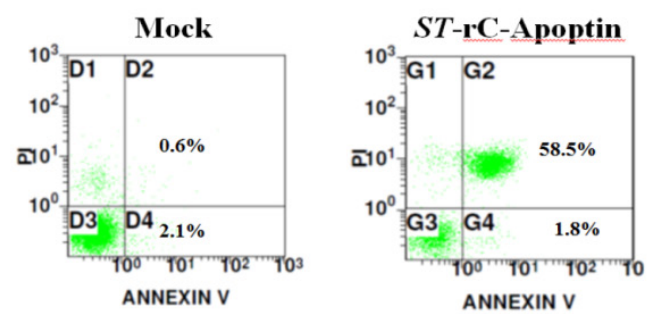

C

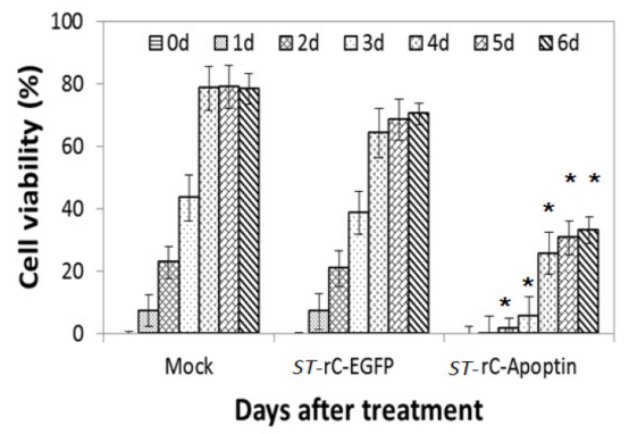

totoxicity following ST-rC-Apoptin infection as compared to infection with ST-rC-EGFP in Hep-2 cells $(\mathrm{p}<0.01)$.

A

B
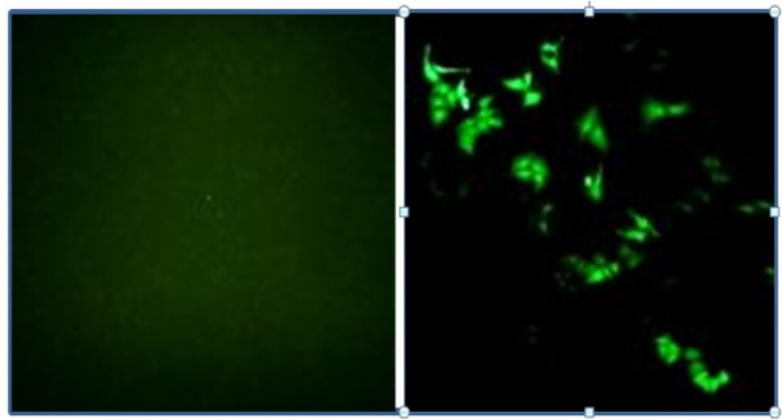

C

Mock $S T$-rC-EGFP $S T$-rC-Apoptin

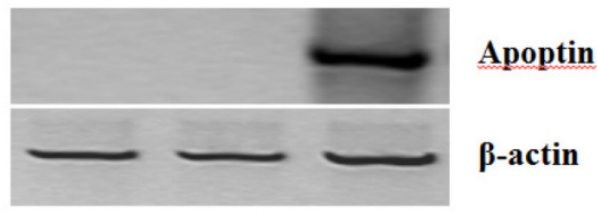

Figure I. Recombinant S. typhimurium mediated expression of Apoptin. Hep-2 cells were infected with ST-rC-EGFP and were observed with fluorescence microscopy 24 hours after infection. (A). non-infected cells (mock). (B). ST-rC-EGFP infected cells (200x magnifications). (C). Western blotting analysis of recombinant $S$. typhimurium-mediated Apoptin (ST-rC-Apoptin) in Hep-2 cells. The cells were harvested at 24 hours after infection.

Figure 2. S. typhimurium-mediated Apoptin induced apoptosis and reduced viability of Hep-2 cells. (A).ST-rC-Apoptin induced apoptosis in Hep-2 cells measured by the increase of Annexin V- PI positive cells. (A: apoptotic cells; V: viable cells). (B). Flow cytometry analysis showed viable, early and late apoptotic cells. Data was presented as mean \pm S.E, $n=2,{ }^{*} p<0.05$. (C). MTT assays indicated that tumour cell viability was significantly reduced with ST-rC-Apoptin treatment. Data was presented as mean \pm S.E, $n=3,{ }^{*} p<0.05$. 


\section{S. typhimurium-mediated Apoptin increases the activity of caspase- 3 and involves Bax, caspase-9 and Cytochrome c}

To elucidate the molecular mechanisms underlying the ST-rC-Apoptin induced apoptosis, caspase-3 and caspase- 8 activities were measured. As observed in Figure 3A, the increase in apoptosis was associated with activation of caspase-3, with 2.7-fold higher than that in the mock cells at 48 hours post-infection. In contrast, caspase- 8 experienced a minimal change following ST-rC-Apoptin infection. Western blot conducted at 48 hour post-infection demonstrated a significant increase in cytochrome $c$, which in turn was associated with an increase in cleaved casapse-9 and Bax protein in ST-rC-Apoptin infected cells, Bcl-2 yet did not show the change (Figure 3B).

A

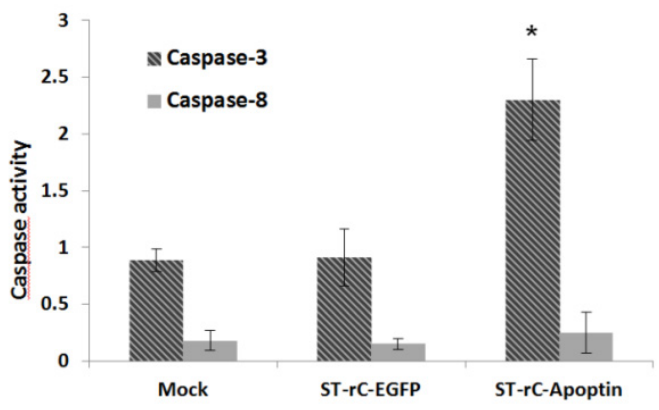

B

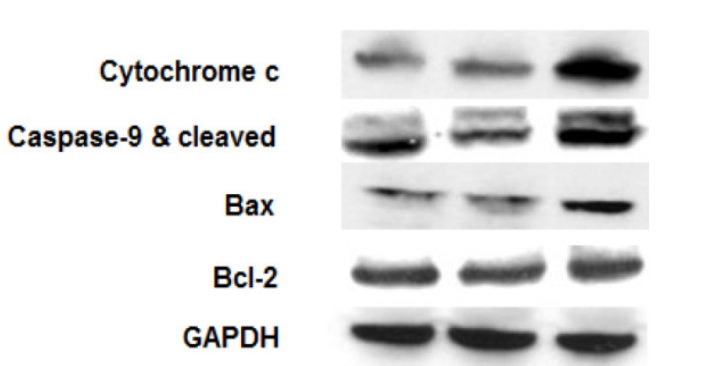

Figure 3. S. typhimurium-mediated Apoptin increased the activity of caspase- 3 and involved in Bax, cleaved caspase- 9 and Cytochrome $c$ expression. (A). Caspase $3 / 8$ activity was measured at 48 hours post-infection with either ST-rC-Apoptin or ST-rC-EGFP compared with mock-infected cells. Data was presented as mean $\pm \mathrm{SE}$; at least two independent experiments conducted in triplicate; ${ }^{*} p<0.05$. (B). Western blotting for Cytochrome c, $\mathrm{Bcl}-2$, caspase- 9 (cleaved caspase-9) and Bax protein expression in Hep-2 cells at 48 hours post-infection. $n=3$.

\section{Systemic administration of S. typhimuri- um-mediated Apoptin effectively inhibits tu- mour growth in Hep-2 xenograft}

To evaluate the therapeutic effects of $S$. typhimurium-mediated Apoptin in human laryngeal cancer, Hep-2 cells were injected into BALB/c nude mice subcutaneously. Once the tumour reached treatment size, ST-rC-Apoptin was then administered intrave- nously. As shown in Figure 4A, a single injection of $1 \times 10^{7} \mathrm{cfu}$ ST-rC-Apoptin showed a trend of tumour growth reduction compared to mock treated mice which received saline. To estimate the survival over time after the treatments for ST-rC-Apoptin and mock groups, A Kaplan-Meier survival curve was generated to test the differences in survival between those two groups. Similarly, the survival curve (Figure 4B) showed a borderline $p$ value $(p=0.06$, log-rank test). Re-injection of the bacteria caused significant tumour regression and prolonged the mice survival from 22 days (mock group) to more than 32 days (ST-rC-Apoptin treated group) (Kaplan-Meier plots of survival, Log-rank test, $\mathrm{p}=0.03$ ) (Figure $4 \mathrm{D}$ ). Interestingly, when tumour volumes were compared with each other on day 20 of the experiment (dash line), the average tumour volume for the ST-rC-Apoptin group $\left(\mathrm{n}=4\right.$ mice) was $500 \mathrm{~mm}^{3}$. Compared with the tumour volume of $750 \mathrm{~mm}^{3}$ for ST-rC-EGFP group ( $\mathrm{n}=2$ mice) and $1200 \mathrm{~mm}^{3}$ for the mock group ( $\mathrm{n}=2$ mice) (Figure $4 \mathrm{C}$ ), the data suggests that ST-rC-Apoptin treated group experienced significant tumour growth delay.

To further quantify tumour growth rate, the tumour volume doubling time (TDT) and tumour growth delay (TGD) analysis were performed. The treatment with Apoptin-expressing bacteria significantly increased the tumour doubling time (TDT) compared with the saline control regardless of whether it was a single or re-dosing injection $(p<0.05)$ (Figure 5A). Consistently, the tumour growth delay (TGD) for ST-rC-Apoptin, was significantly greater than the saline control treatment $(\mathrm{p}<0.01)$, as well as in the ST-rC-EGFP treated group $(\mathrm{p}<0.05)$. Compared with the single dose group of ST-rC-Apoptin, the redosing group demonstrated a significant increase in TGD from 8 days to 18 days $(\mathrm{p}<0.01$ ) (Figure $5 \mathrm{~B}$ ). Tumour microvessel density was subsequently evaluated through immunohistochemical staining for endothelial-specific antigen CD31. As shown in Figure 5C, the ST-rC-Apoptin treated group showed a significant reduction in CD31 staining and obliteration of the normal vasculature in the tumour area.

\section{Tumour specificity of S. typhimurium -Apoptin}

Previous studies have shown that $S$. typhimurium has the ability to present itself within tumours due to its preferential colonization in nutrient-rich and hypoxic microenvironments $[9,13]$. To study the spatiotemporal dynamics of bacterial distribution within tumours, a syngeneic subcutaneous model of laryngeal carcinoma was investigated. A single systemic inoculation of $1 \times 10^{7} \mathrm{cfu}$ ST-rC-Apoptin resulted in the preferential colonization of bacteria within the tumour at a 1,000-fold increase compared to liver tissue. Repeated intravenous doses of ST-rC-Apoptin were 
also well tolerated and maintained the same pattern of distribution (Figure 6A). Systemic toxicity of $S$. typhimurium-Apoptin was evaluated by Hematoxylin and Eosin staining in the major organs of the mice. No significant side effects were observed in both single and re-dosing treatments (Figure 6B).
A.

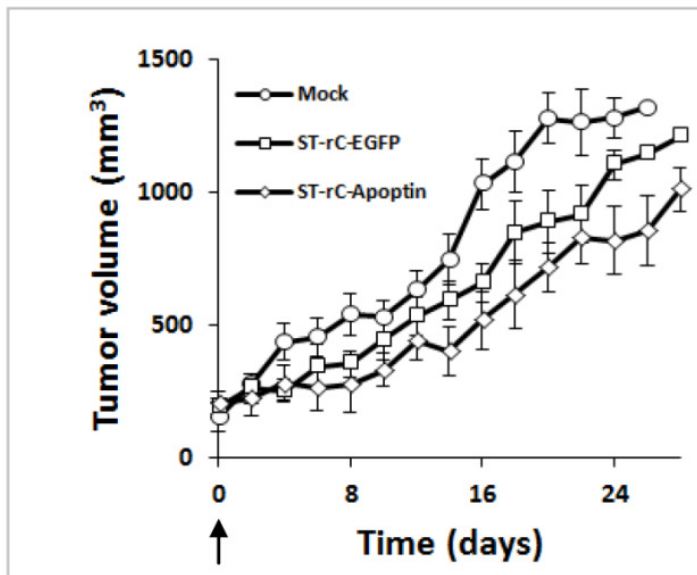

C.

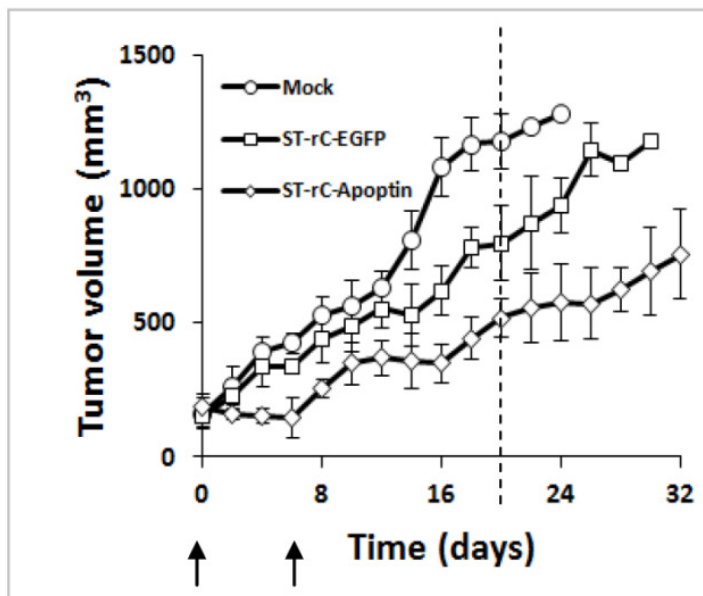

B.

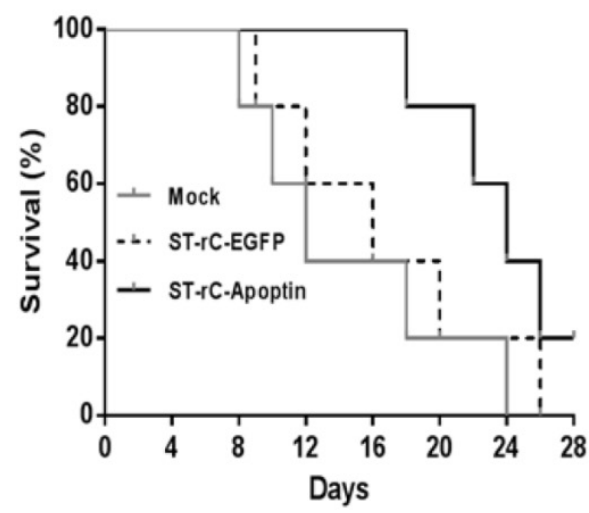

D.

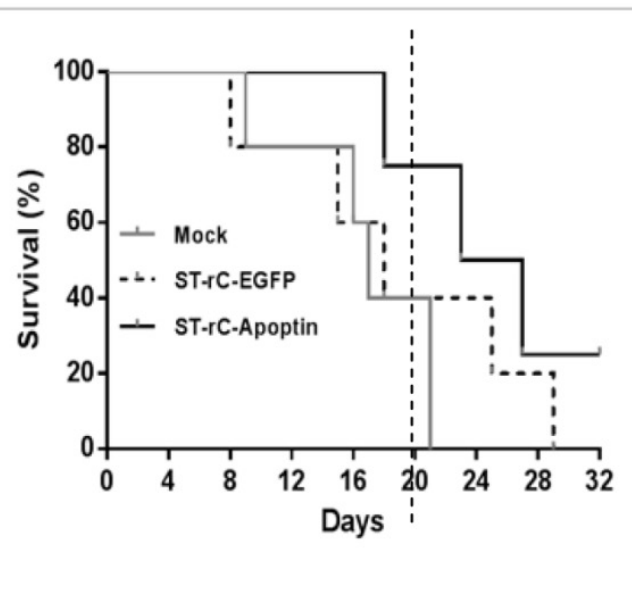

Figure 4. Systemic administration of $S$. typhimurium-mediated Apoptin effectively inhibited tumour growth in Hep-2 xenograft. (A). A single injection of $1 \times 10^{7} \mathrm{cfu} S T$-rC-Apoptin showed a trend of tumour growth reduction in comparison to mock treated mice; $\mathrm{n}=5$ mice/group. (B). Kaplan-Meier plots of survival analysis. Data was analyzed using the log-rank test with $p=0.06$. (C) and (D). Repeated injections of ST-rC-Apoptin significantly regressed tumour growth and prolonged mice survival; $n=5$ mice/group, $p=0.03$. Each dot on the graphs Figure $A$ and $C$ presented an averaged value of tumour size from surviving mice at each measuring time. Data was analyzed by log-rank test and was presented as mean \pm SE; Error bar, standard error; no error bars were available at the end of the experiment due to the death of mice. Arrows - time of bacteria injection; Dash line - a representative time to compare different tumour sizes in different treatment groups. 
A.

Figure 5. Tumour growth delay and microvessel density involvement. (A). Tumour doubling times significantly increased through treatment with ST-rC-Apoptin regardless of whether it was a single or re-dosing injection. Data was presented as mean $\pm \mathrm{SE}$, $\mathrm{n}=5$ mice/group, $* \mathrm{p}<0.05$. (B). Significant tumour growth delay $(2.2$ fold $)$ in the re-dosing treatment group. Data was presented as mean $\pm \mathrm{SE}, \mathrm{n}=5$ mice/group; $* *$ p $<0.01$. (C). Tumour microvessel density was measured in both ST-rC-EGFP (a) and ST-rC-Apoptin (b) infection groups.

(1)

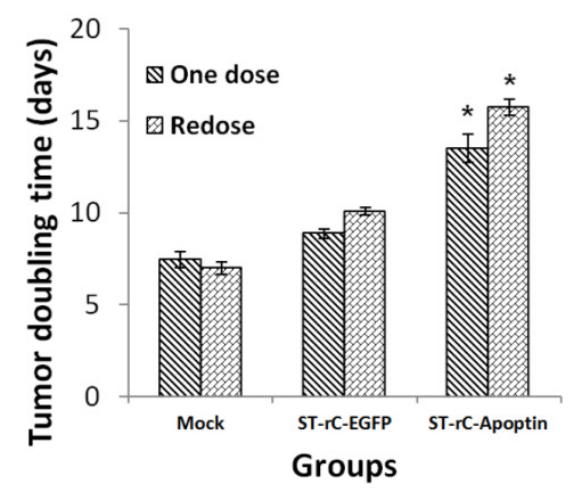

C.

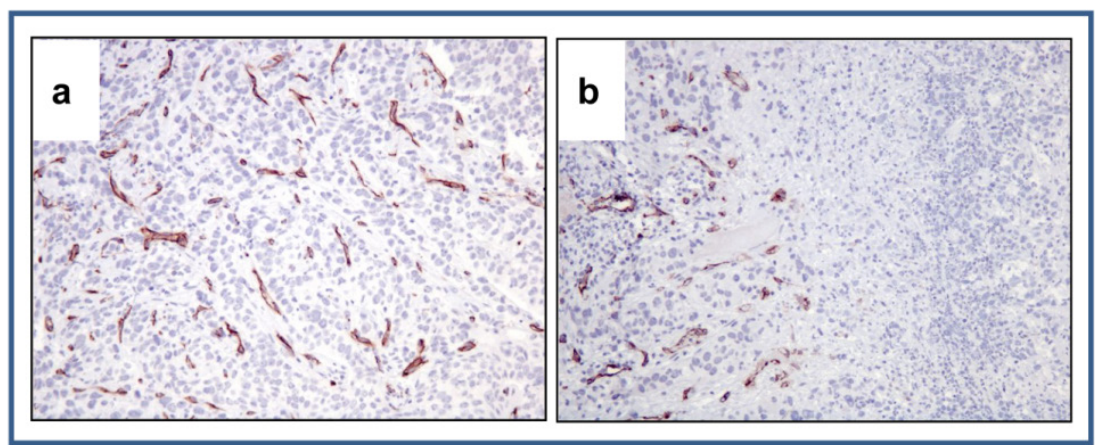

B.

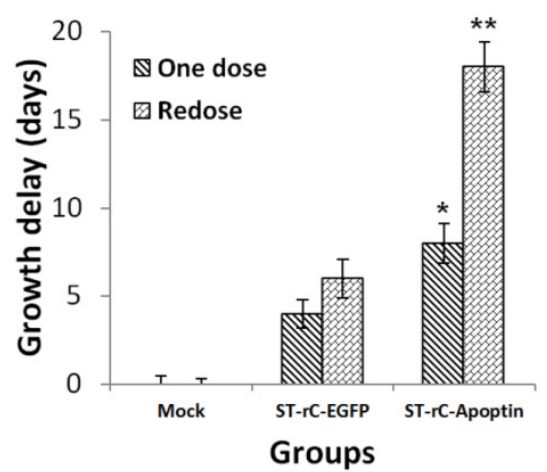

A.

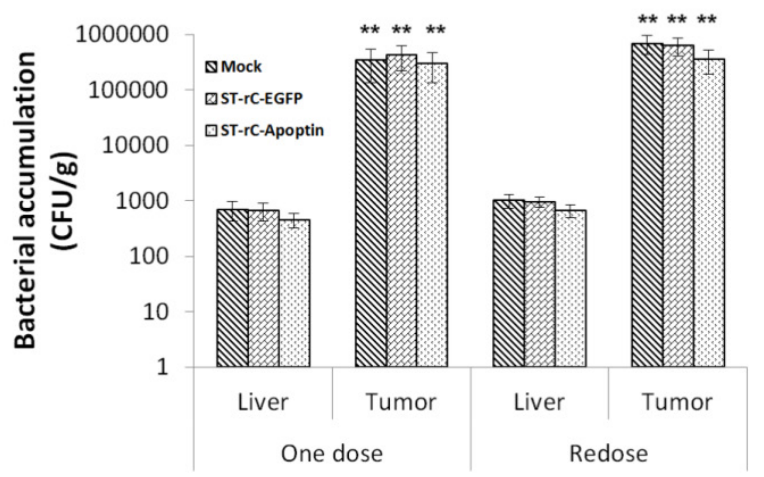

B.

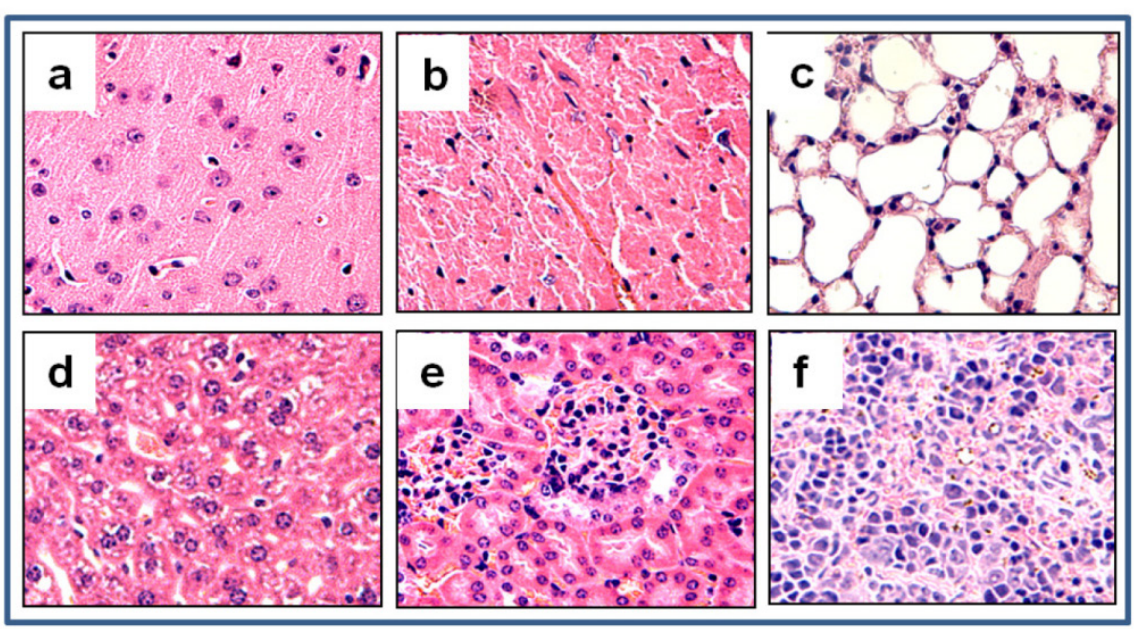

Figure 6. Tumour specific distribution of $S$. typhimurium-mediated Apoptin. (A). Systemic inoculation of $1 \times 10^{7} \mathrm{cfu}$ ST-rC-Apoptin resulted in the preferential colonization of bacteria within the tumour at a 1,000-fold increase compared to liver tissue in both single and redosing treatment. Data was presented as mean $\pm S E, n=5$ mice/group. (B). Histological staining in the major organs of the mice. a. Brain; b. Heart; c. Lung; d. Liver; e. Kidney; f. Spleen. 


\section{Discussion}

This is the first report utilizing a recombinant attenuated Salmonella typhimurium to express tumour-specific apoptosis-inducing gene, Apoptin, in human laryngeal cancer. Salmonella typhimurium has been used before as a therapeutic delivery vector because of its natural tropism and immune privilege [24, 25]. Particularly, Salmonella typhimurium itself can secrete and inject more than 20 effector proteins into host cytosol/lysosome, which can inhibit phagosome-lysosome fusion and interact with the host machinery to allow the bacteria to survive and replicate in host cells [26]. Notably, the attenuated S. typhimurium strains possess anti-tumour activity through the expression of some genes encoding therapeutic proteins [27, 28]. A previous study indicated that the delivery of TRAIL and VP3 genes by attenuated $S$. typhimurium in gastric cancer cells achieve significant tumour inhibition through intra-tumour injection [29]. More recently, Blache CA at al. further demonstrated that systemic administration of the clinically relevant S. typhimurium strain, VNP20009, carrying an indoleamine 2,3-dioxygenase 1-specific shRNA plasmid results in tumour growth suppression in aggressive melanomas [30]. Thus, it is attractive to explore the use of combining the attenuated S. typhimurium and Apoptin gene for therapeutic applications. We have previously demonstrated a combinative anti-tumour effect in human laryngeal carcinoma cells using a nucleic vaccine consisting of pVVP3IL-18HN expressing Apoptin gene, Newcastle disease virus HN gene and IL-18 gene [31]. This platform allowed us to successfully generate an Apoptin expression vector that effectively delivered Apoptin into laryngeal cancer cells (Figure 1C). The results presented in this study provide important evidence that attenuated $S$. typhimurium-mediated Apoptin infection can induce significant cytotoxicity, and cell apoptosis in Hep-2 laryngeal carcinoma cells. Furthermore, intravenous delivery of attenuated $S$. typhimurium-mediated Apoptin decreases tumour growth, reduces tumour microvessel density and prolongs the survival of mice.

The accurate mechanism of Apoptin-induced cell death has not yet been fully elucidated. A fundamental apoptotic pathway for Apoptin is independent of p53 and requires caspase activation [18]. The intrinsic pathway is involved in the release of cytochrome $\mathrm{c}$ from the damaged mitochondrion to the cytosol, which further leads to the activation of caspase-9. Caspase-9 then triggers a cascade of caspases, e.g. caspase- 3 and caspase-7, which in turn results in morphological and biochemical changes associated with apoptosis [32, 33]. Our current study demonstrates a strong apoptotic induction rate $(60 \%)$ by Apoptin (Figure 2A and 2B). This was mediated by the elevation of both cytochrome $\mathrm{C}$ and cleaved caspase-9, resulting in the activation of caspase- 3 in laryngeal cancer cells (Figure 3A and 3B).

The pro- and anti-apoptotic Bcl-2 protein family plays an important role in the presence of Apoptin. Burek $\mathrm{M}$ et al. reported that Bcl-2 and Bcl-xL inhibit Apoptin-induced cell death, whereas Bax expression sensitizes cells to apoptosis [34]. It is known that Bcl-2 binds to and inactivates Bax and other pro-apoptotic proteins, thereby inhibiting apoptosis [29, 35]. Our findings show that Bax protein is overexpressed in ST-rC-Apoptin treated cells, leading to the activation of cytochrome c, while Bcl-2 expression does not change after the infection, suggesting that Apoptin-induced apoptosis is independent of Bcl-2, at least in the regulation of laryngeal cancer cells (Figure 3B). Given the complexity of the apoptotic pathway, Apoptin-induced cell death can also occur in a caspase independent manner. DNA damage response (DDR) signaling is also required to induce Apoptin nuclear localization in primary cells [36]. Additionally, the cytoplasmic translocation of Nur77 is crucial for the toxicity of Apoptin [37]. Moreover, Akt-triggered activation of CDK 2 and PKC kinase have been identified as key effectors for Apoptin phosphorylation [38].

Many apoptosis-related cancer gene therapy studies use combined approaches, by incorporating conventional therapies (such as RT and chemotherapy) with a combination of therapeutic genes [36], or by utilizing a fusion protein [39]. To increase clinical benefits, it is important to evaluate the efficacy and safety. In our study, attenuated S. typhimurium and Apoptin induce apoptosis synergistically and enhance growth inhibition of laryngeal cancer. Tumour growth delay was observed with a 2.2-fold increase through the repeated administration of ST-rC-Apoptin, resulting in a significant survival benefit and decreased normal tissue toxicity (Figure 5B; Figure 4D and Figure 6B). Previous studies have investigated the mechanisms of $S$. typhimurium accumulation in tumours. S. typhimurium was found to penetrate into tumours aggressively with $200 \sim 10,000$-fold more than any other organs $[13,40]$. Consistent with previous work, our results also demonstrate that there were approximately 1,000 fold higher accumulation of ST-rC-Apoptin in the tumour when compared to the liver. Furthermore, the additive administration of ST-rC-Apoptin continuously preserved the bacteria level in tumours (Figure 6A), indicating that the Apoptin-expressing bacteria have specific tumour selectivity and could be considered as a powerful tool for the treatment of laryngeal cancer. 
The investigation of safety and toxicity by histological staining confirmed that there were no acute hepatic, lung and kidney adverse effects after systemic injection of $1 \times 10^{7} \mathrm{cfu}$ bacteria (Figure 6B). More interestingly, tumour angiogenesis reduction was only observed in the ST-rC-Apoptin treated group. Our results suggest a synergistic effect by which $S$. typhimurium accumulates into regions between viable tumour and necrosis, releasing Apoptin, leading to local tumour cell death and decreased tumour microvessels. However, further investigation is needed to obtain a better understanding of the complex interaction between the tumour microenvironment and Apoptin.

In conclusion, our study presents the first tumour-specific therapeutic use of attenuated Salmonella typhimurium mediated Apoptin in human laryngeal cancer. It shows a significant inhibition of tumour cell growth, both in vitro and in vivo, through regulation of apoptotic signaling pathways involving Bax, cytochrome c, caspase- 9 and caspase-3. Systemic delivery of attenuated Salmonella typhimurium mediated Apoptin has synergistic activity which enhances tumour growth delay and causes tumour microvessels reduction with minimum toxicity to normal tissues. This underscores the promising potential for the use of Salmonella typhimurium mediated Apoptin as a therapeutic option for laryngeal cancer.

\section{Acknowledgments}

The authors acknowledge the financial support of the National Natural Science Foundation of China (81072210) and the Science and Technology R\&D program of Jilin Province, China (201015166). We would also like to thanks Dr. Xue-jian Zhao of Institute of Basic Medical Sciences, Jilin University for his generous supports for attenuated $S$. typhimurium phoP/phoQ null strain LH430.

\section{Competing Interests}

The authors have declared that no competing interest exists.

\section{References}

1. Wong TS, Gao W, Li ZH, Chan JY, Ho WK. Epigenetic dysregulation in laryngeal squamous cell carcinoma. Journal of oncology. 2012;: 739461.

2. Jemal A, Bray F, Center MM, Ferlay J, Ward E, Forman D. Global cancer statistics. CA: a cancer journal for clinicians. 2011;61: 69-90.

3. Chu EA, Kim YJ. Laryngeal cancer: diagnosis and preoperative work-up. Otolaryngologic clinics of North America. 2008; 41: 673-95.

4. Freedman ND, Abnet CC, Leitzmann MF, Hollenbeck AR, Schatzkin A. Prospective investigation of the cigarette smoking-head and neck cancer association by sex. Cancer. 2007; 110: 1593-601.

5. Hoffmann TK. [Systemic therapy strategies for head-neck carcinomas: current status]. Laryngo- rhino- otologie. 2012;91 Suppl 1: S123-43.

6. Forbes NS. Profile of a bacterial tumour killer. Nat Biotechnol. 2006; 24: 1484-5. doi:nbt1206-1484[pii] 10.1038/nbt1206-1484.

7. Ryan RM GJ, Lewis CE. Use of bacteria in anti-cancer therapies. Bioessays. 2006; 28(1):84-94.
8. Nemunaitis J, Cunningham C, Senzer N, Kuhn J, Cramm J, Litz C, et al. Pilot trial of genetically modified, attenuated Salmonella expressing the E. coli cytosine deaminase gene in refractory cancer patients. Cancer gene therapy. 2003; 10: 737-44.

9. Ganai S, Arenas RB, Forbes NS. Tumour-targeted delivery of TRAIL using Salmonella typhimurium enhances breast cancer survival in mice. British journal of cancer. 2009; 101: 1683-91.

10. Jain RK, Forbes NS. Can engineered bacteria help control cancer? Proceedings of the National Academy of Sciences of the United States of America. 2001; 98: 14748-50.

11. Low KB, Ittensohn M, Le T, Platt J, Sodi S, Amoss M, et al. Lipid A mutant Salmonella with suppressed virulence and TNFalpha induction retain tumour-targeting in vivo. Nature biotechnology. 1999; 17: 37-41.

12. Kasinskas RW, Forbes NS. Salmonella typhimurium specifically chemotax and proliferate in heterogeneous tumour tissue in vitro. Biotechnology and bioengineering. 2006; 94: 710-21.

13. Forbes NS, Munn LL, Fukumura D, Jain RK. Sparse initial entrapment of systemically injected Salmonella typhimurium leads to heterogeneous accumulation within tumours. Cancer research. 2003; 63: 5188-93.

14. Kasinskas RW, Forbes NS. Salmonella typhimurium lacking ribose chemoreceptors localize in tumour quiescence and induce apoptosis. Cancer research. 2007; 67: 3201-9.

15. Tredan O, Galmarini CM, Patel K, Tannock IF. Drug resistance and the solid tumour microenvironment. Journal of the National Cancer Institute. 2007; 99: 1441-54.

16. Danen-Van Oorschot AA, Fischer DF, Grimbergen JM, Klein B, Zhuang $\mathrm{S}$, Falkenburg $\mathrm{JH}$, et al. Apoptin induces apoptosis in human transformed and malignant cells but not in normal cells. Proceedings of the National Academy of Sciences of the United States of America. 1997; 94: 5843-7.

17. Leliveld SR, Zhang YH, Rohn JL, Noteborn MH, Abrahams JP. Apoptin induces tumour-specific apoptosis as a globular multimer. The Journal of biological chemistry. 2003; 278: 9042-51.

18. Zhuang SM, Shvarts A, van Ormondt H, Jochemsen AG, van der Eb AJ, Noteborn $\mathrm{MH}$. Apoptin, a protein derived from chicken anemia virus, induces p53-independent apoptosis in human osteosarcoma cells. Cancer research. 1995; 55: 486-9.

19. Backendorf $C$, Visser $A E$, de Boer $A G$, Zimmerman $R$, Visser $M$, Voskamp P, et al. Apoptin: therapeutic potential of an early sensor of carcinogenic transformation. Annual review of pharmacology and toxicology. 2008; 48: 143-69.

20. Pavet V, Portal MM, Moulin JC, Herbrecht R, Gronemeyer H. Towards novel paradigms for cancer therapy. Oncogene. 2011;30: 1-20.

21. Zhang L, Gao L, Zhao L, Guo B, Ji K, Tian Y, et al. Intratumoural delivery and suppression of prostate tumour growth by attenuated Salmonella enterica serovar typhimurium carrying plasmid-based small interfering RNAs. Cancer research. 2007; 67: 5859-64.

22. Su J, et al. Inhibition of Dual Specific Oncolytic Adenovirus on Esophageal Cancer via Activation of Caspases by a Mitochondrial-dependent Pathway. Chem Res Chinese Universities. 2012; 28(3): 465-71.

23. Kleinbaum DG, Klein M. Survival Analysis. A self - learning text. Springier; 2005

24. Lee $\mathrm{CH}$. Engineering bacteria toward tumour targeting for cancer treatment: current state and perspectives. Applied microbiology and biotechnology. 2012;93: 517-23.

25. Paterson Y, Guirnalda PD, Wood LM. Listeria and Salmonella bacterial vectors of tumour-associated antigens for cancer immunotherapy. Seminars in immunology. 2004;22: 183-9.

26. Chantal G. Forest EF, Sebastien C. Sabbagh and Elyse Ferraro, Intracellular survival of Salmonella enterica serovar Typhi in human macrophages is independent of Salmonella pathogenicity island (SPI)-2. Microbiology. 2010; 156: 3689-98.

27. Agorio C, Schreiber F, Sheppard M, Mastroeni P, Fernandez M, Martinez MA, et al. Live attenuated Salmonella as a vector for oral cytokine gene therapy in melanoma. The journal of gene medicine. 2007; 9: 416-23.

28. Ryan RM, Green J, Williams PJ, Tazzyman S, Hunt S, Harmey JH, et al. Bacterial delivery of a novel cytolysin to hypoxic areas of solid tumours. Gene therapy. 2009; 16: 329-39.

29. Cao HD, Yang YX, Lu L, Liu SN, Wang PL, Tao XH, et al. Attenuated Salmonella typhimurium carrying TRAIL and VP3 genes inhibits the growth of gastric cancer cells in vitro and in vivo. Tumouri. 2010;96: 296-303.

30. Blache CA, Manuel ER, Kaltcheva TI, Wong AN, Ellenhorn JD, Blazar BR, et al. Systemic delivery of Salmonella typhimurium transformed with IDO shRNA enhances intratumoural vector colonization and suppresses tumour growth. Cancer research. 2012; 72: 6447-56.

31. Guan GF JN, Mi ZQ, Li X, Lian H, Jin CS, Sun LL, Wen LJ. Construction of the nucleic vaccine pVVP3L-18HN and its antitumour effect on 
human laryngeal carcinoma. Zhonghua Er Bi Yan Hou Tou Jing Wai Ke Za Zhi 2005; 40(8).

32. Argiris K, Panethymitaki C, Tavassoli M. Naturally occurring, tumour-specific, therapeutic proteins. Experimental biology and medicine. 2012;236: 524-36.

33. Tavassoli M, Guelen L, Luxon BA, Gaken J. Apoptin: specific killer of tumour cells? Apoptosis. 2005; 10: 717-24.

34. Burek M MS, Burek CJ, Daniel PT, Schulze-Osthoff $K$, Los M. Apoptin-induced cell death is modulated by Bcl-2 family members and is Apaf-1 dependent. Oncogene. 2006; 25(15): 2213-22.

35. Flora Tzifi CE, et al. The Role of BCL2 Family of Apoptosis Regulator Proteins in Acute and Chronic Leukemias. Advances in Hematology. 2012.

36. Shinoura N, Saito K, Yoshida Y, Hashimoto M, Asai A, Kirino T, et al. Adenovirus-mediated transfer of bax with caspase- 8 controlled by myelin basic protein promoter exerts an enhanced cytotoxic effect in gliomas. Cancer gene therapy. 2000; 7: 739-48.

37. Maddika S, Booy EP, Johar D, Gibson SB, Ghavami S, Los M. Cancer-specific toxicity of apoptin is independent of death receptors but involves the loss of mitochondrial membrane potential and the release of mitochondrial cell-death mediators by a Nur77-dependent pathway. Journal of cell science. 2005; 118: 4485-93.

38. Maddika S, Panigrahi S, Wiechec E, Wesselborg S, Fischer U, Schulze-Osthoff $\mathrm{K}$, et al. Unscheduled Akt-triggered activation of cyclin-dependent kinase 2 as a key effector mechanism of apoptin's anticancer toxicity. Molecular and cellular biology. 2009; 29: 1235-48.

39. Lee MS, Sun FC, Huang CH, Lien YY, Feng SH, Lai GH, et al. Efficient production of an engineered apoptin from chicken anemia virus in a recombinant E. coli for tumour therapeutic applications. BMC biotechnology. 2012;12: 27.

40. Sorenson B, et al. Safety and immunogenicity of Salmonella typhimurium expressing C-terminal truncated human IL-2 in a murine model. Biologics 2010; 4: 61-73. 